\title{
Analysis of Hadron Models to Better Predict Neutrino Flux for DUNE
}

Ethan Tuttle, Ball State University, SIST Intern

\section{Introduction}

My project was to characterize the interactions of two hadron production models QGSP_BERT and FTFP_BERT. Then I compared them with experimental data, the data used was from NA49. NA49 was an experiment conducted at CERN, NA49 tested the particle production of various hadronic interactions on targets of different materials. QGSP_BERT is being used to simulate the LBNF beamline, so it is important for DUNE that the QGSP_BERT model is characterized. Because then predictions of the neutrino flux are better understood and can have more accurate uncertainties so measurements of neutrino oscillation can be made more reliably and accurately.

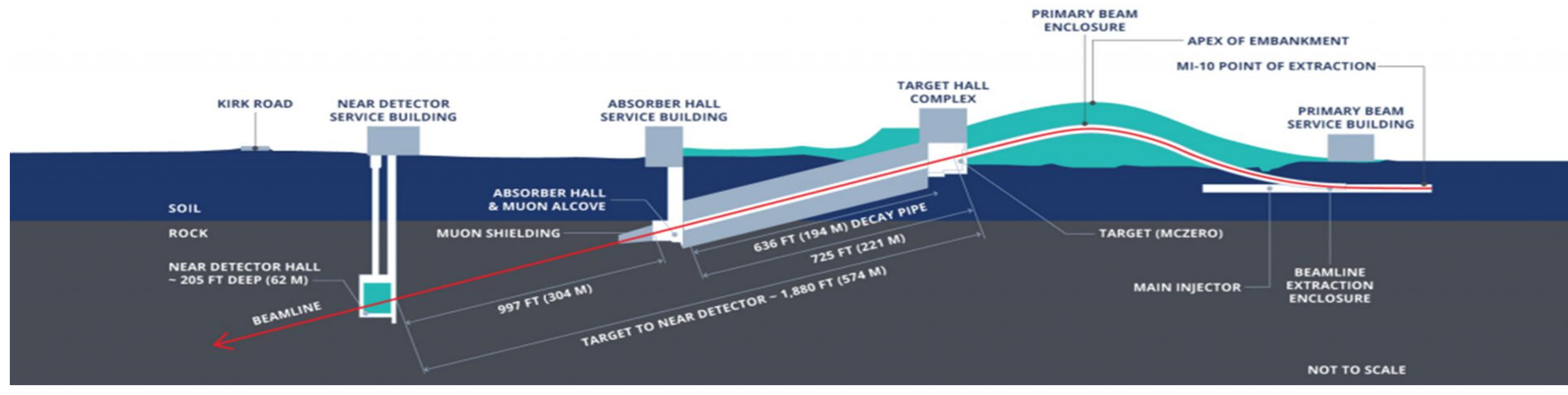

Methods

The data was generated using two Geant4 programs one called G4LBNF, which simulates the LNBF beamline. The other program is called G4HP. G4HP is used to obtain the cross-section histograms:

1. Setting the hadron model to use to either QGSP_BERT or FTFP_BERT.

2. Then the geometry and composition of the target are simulated.

3. Next protons at a given energy are fired at the target.

4. The data from the proton on target interaction is stored in a ROOT TTree. The material used in the target for DUNE is carbon, so the interactions analyzed for my project were proton on carbon. Proton on carbon interactions create particles such as pions and kaons. To get the cross-section data from the simulation a program called CreateYields is used to get the production yields of the particles created. Then the output from the CreateYields program is used in another program called CreatelnvXS to create histograms of the invariant cross section of the interaction for a particular particle e.g. positively charged pions. The reason we use this method is because the data for the cross section is not directly available.

QGSP_BERT Pion Momentum

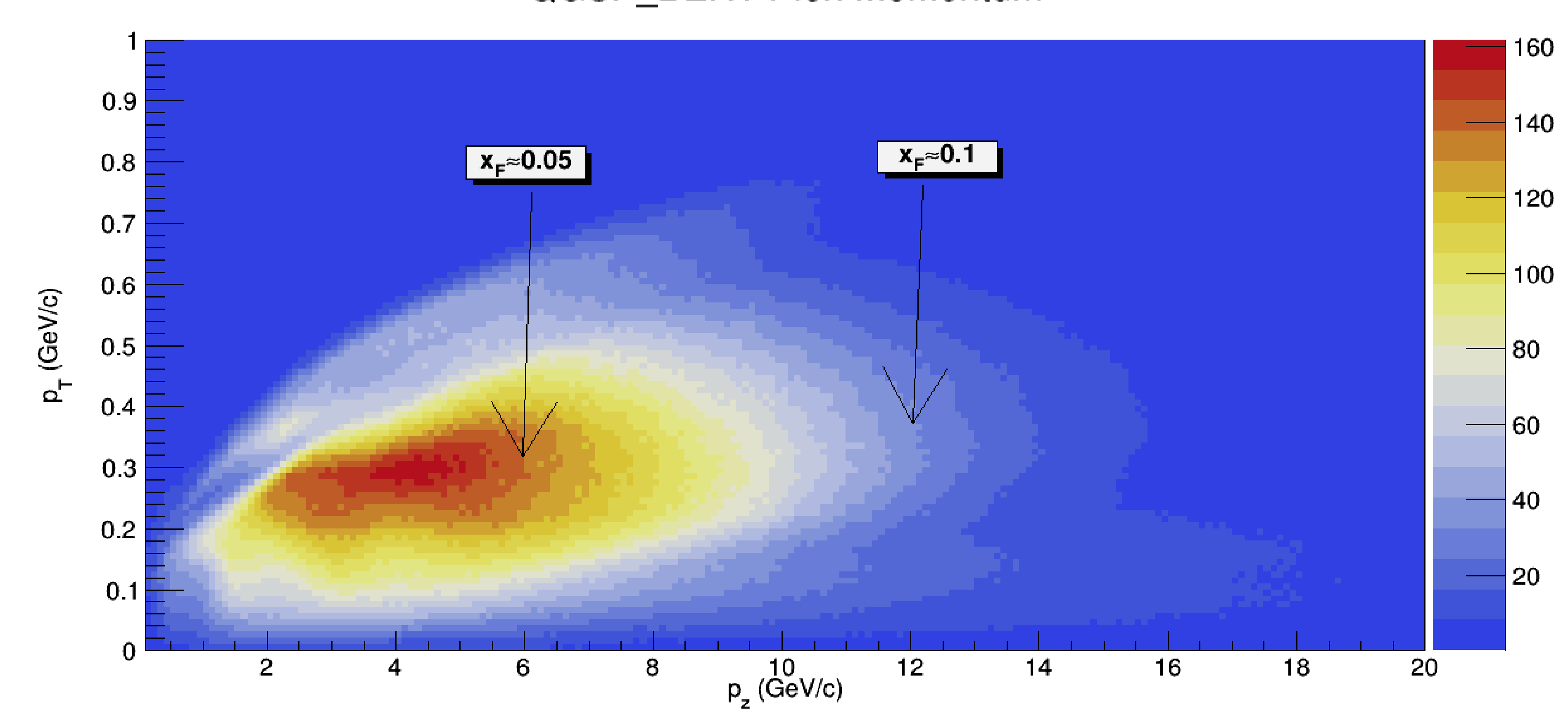

Results and Discussion

The results of the simulation show that for both positive and negative pions at low transverse momentum QGSP_BERT has a larger cross section compared to NA49. QGSP_BERT's cross section also generally decreases as the transverse momentum increases until the cross section for QGSP_BERT is smaller than the cross section for NA49. On the other hand FTFP_BERT usually starts close to the NA49 data when transverse momentum is low and at higher transverse momentum it overproduces compared to NA49. Also of note is that the cross section of positive pions is greater than the cross section for negative pions. Important to know because the LBNF has magnets that can focus charged particles we want, and defocus charged particle we don't want. However, there will still be some background of the particles we don't want that get past the magnets into the beamline.
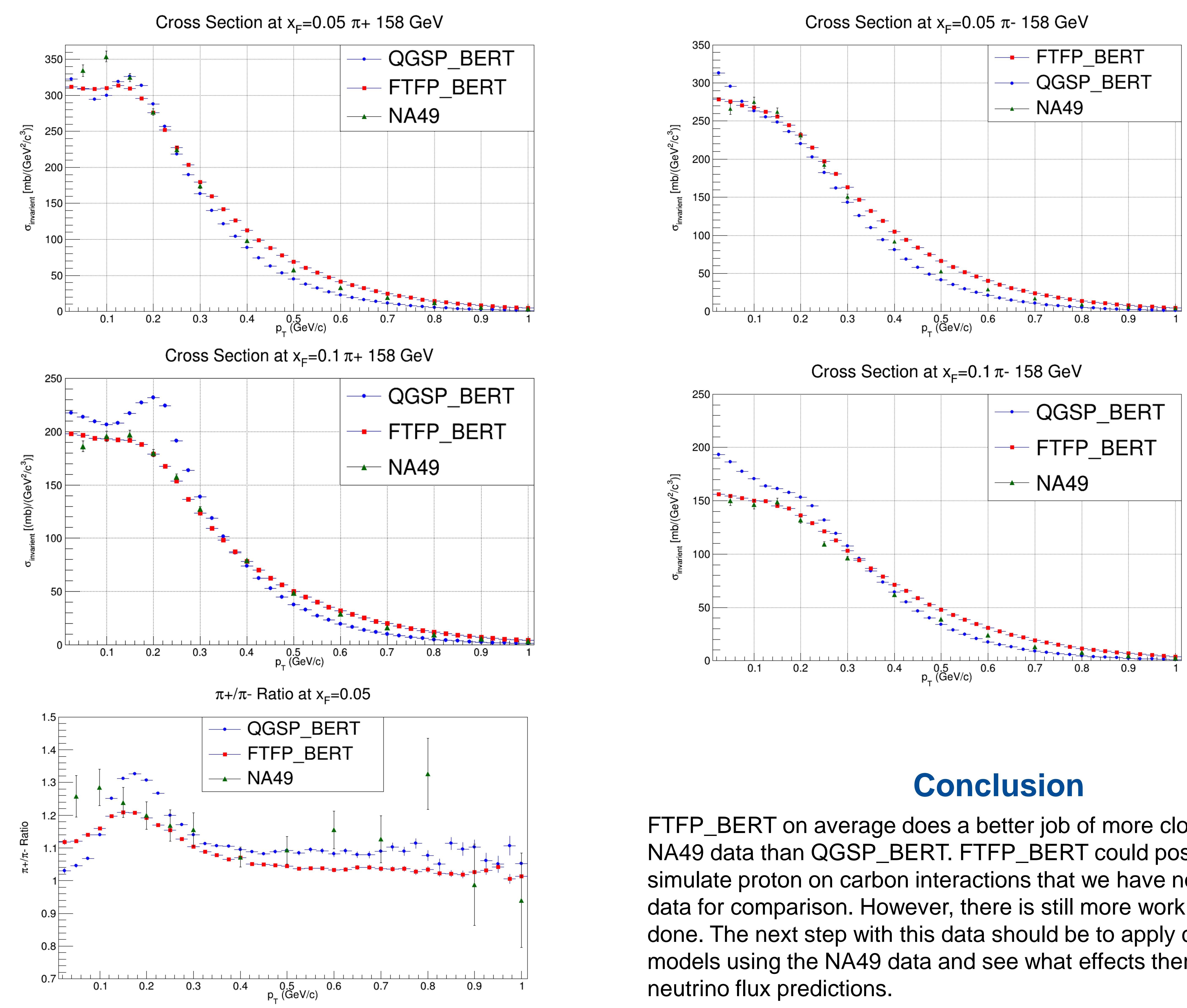

Conclusion

FTFP BERT on average does a better job of more closely following the NA49 data than QGSP BERT. FTFP BERT could possibly be used to simulate proton on carbon interactions that we have no experimental data for comparison. However, there is still more work that needs to be data for comparison. However, there is still more work that needs to be
done. The next step with this data should be to apply corrections to the models using the NA49 data and see what effects there are on the neutrino flux predictions.

\section{Acknowledgements}

First and foremost I would like to thank my supervisor Leo Aliaga for guiding me through this project and for being willing to teach me more about programming and physics. I would like to thank my mentors Arden Warner and Charlie Orozco for all the help and advice they have provided. I also want to thank the SIST committee for their hard work in creating this online internship experience. This manuscript has been authored by Fermi Research Alliance, LLC under Contract No. DE-AC02-07CH11359 with the U.S. Department of Energy, Office of Science, Office of High Energy Physics. 